\title{
«To those who don't deserve the truth, don't give it». Sobre la posibilidad de ficcionalización de lo Real y sus secuelas en The Shawl de Cynthia Ozick
}

\author{
Ramón Soto GÁmEZ \\ Universidad Complutense de Madrid \\ ramsoto@ucm.es
}

\begin{abstract}
RESUMEN
Los dos relatos que conforman The Shawl retratan un episodio atroz de los campos de exterminio («The Shawl») y la alienación posterior de una superviviente («Rosa»). Aunque puedan partir de materiales históricos, ambos asuntos se reelaboran desde planteamientos ficcionales. La propuesta pretende indagar en la legitimidad ética y posibilidad de la plasmación estética del Holocausto y sus consecuencias. Frente al rigor historicista, la literatura debería ser capaz de comunicar y hacer comprender, si no lo que ocurrió, sí aquello que pudo haber ocurrido, si bien, cuando lo Real se resiste a ser constreñido por la palabra, ni siquiera la ficción puede develar de aquello que se resiste a la representación.
\end{abstract}

Palabras clave: ficciones del Holocausto, indecibilidad y representación de lo Real, Cynthia Ozick.

«To those who don't deserve the truth, don't give it». On the possibility to fictionalize the Real and its sequels in Cynthia Ozick's The Shawl

\begin{abstract}
The two short stories included in The Shawl show the atrocity of the death camps («The Shawl») and a survivor's subsequent alienation («Rosa»). Though built upon historic materials, both themes are reworked from a fictional perspective. The paper will try to discern the ethical legitimacy and possibility of an aesthetical representation of the Holocaust and its consequences. Facing the rigorism of History, Literature should be able to communicate and make understandable whatever it could have happened, if not what really happened. But whenever the Real resists to be expressed in words, not even the fiction can reveal that which resists any kind of representation.
\end{abstract}

Keywords: fictions of the Holocaust, untellibility and representation of the Real, Cynthia Ozick.

Frente al estatus crítico del que gozan las historias cortas «The Shawl» (1980) y «Rosa» (1983), originalmente publicadas en The New Yorker y editadas de manera conjunta con el título de la primera de ellas en 1989, galardonadas ambas con el Pre- 
mio O. Henry el año siguiente a su publicación, sorprende el escaso interés que la obra de Cynthia Ozick ha suscitado en ámbito hispano, donde la edición que de $E l$ chal hizo Montesinos (1991) lleva décadas descatalogada. Mientras el National Endowment for the Arts editó en Estados Unidos una guía sobre The Shawl para su difusión en bibliotecas y centros de enseñanza, en España, de acuerdo con las bases de datos académicas, apenas pueden consignarse dos valedoras de las ficciones de Ozick, Luisa Juárez Hervás - cuya tesis doctoral será citada en estas páginas- y María Jesús Fernández Gil ${ }^{1}$. Este artículo pretende acercarse al tratamiento del Holocausto como materia literaria en los dos relatos de The Shawl, si bien no tanto para justificarlos como posibilidad de acercamiento al hecho histórico como para interponer una distancia crítica entre el hecho (o el testimonio de quienes lo vivieron) y su conversión en objeto estético, proceso de destilación donde no pocas veces se evapora la carga ética que debería ser inherente a cualquier aproximación sobre esta materia.

Para defender la intromisión de la sustancia histórica en la forma literaria, se suele acudir al conocidísimo pasaje de la Poética (1451a-b) que diferencia poesía de historia esgrimiendo que las condiciones de posibilidad de representación de esta son mucho más limitadas que las de aquella, puesto que

no corresponde al poeta decir lo que ha sucedido, sino lo que podría suceder, esto es, lo posible según la verosimilitud o la necesidad. En efecto, el historiador y el poeta no se diferencian por decir las cosas en verso o en prosa [...]; la diferencia está en que uno dice lo que ha sucedido y el otro, lo que podría suceder. Por eso también la poesía es más filosófica que la historia; pues la poesía dice más bien lo general, y la historia, lo particular. (Aristóteles 1999: 157-158)

No obstante, habría que cuestionarse hasta qué punto la ficción puede arrojar alguna luz sobre un hecho histórico que constituye una excepción en la historia de la humanidad y cuya capacidad para ser, no ya comunicado, sino expresado, muestra una tenacidad difícilmente separable de la vivencia. Cabría entonces preguntarse hasta qué punto quienes no estuvimos allí somos merecedores del acceso a esa verdad del superviviente, como hace Rosa en la afirmación que da título a estas páginas, mientras ella misma se engaña escribiendo una carta a Magda -su hija, brutalmente asesinada por un oficial nazi- como si esta hubiese sobrevivido y hubiese desarrollado una vida en el exilio norteamericano (Ozick 1990: 44).

En el tratamiento del Holocausto resultaría posible establecer una gradación según el grado de mediación del relato, que comenzaría con el testimonio in fieri (Diario de Ana Frank, cartas de las víctimas), pasaría por el testimonio - a posteriori-de los supervivientes (la obra de Primo Levi o Elie Wiesel) y la transmisión en

1 Towards a Poetics of Memory: Charlotte Delbo's and Cynthia Ozick's Representation of the Holocaust Experience (tesis doctoral inédita). Universidad de Salamanca, 2011. Habría que añadir el artículo que se citará y sendos capítulos dedicados a Ozick en sus libros Traducir el horror: la intersección de la ética, la ideología y el poder en la memoria del Holocausto (Peter Lang, 2013) y El papel (est)ético de la literatura en la conmemoración del Holocausto (Dykinson, 2014). 
segundo grado de sus relatos (Maus de Art Spiegelmann), y terminaría con la ficción, susceptible asimismo de compartimentarse en diferentes categorías (no parecen equiparables El arca de Schindler y El niño con el pijama de rayas, por poner dos ejemplos). Con excepción del primer grupo de documentos, en los casos restantes se plantea de manera persistente -como un eco perverso de los principios de Gorgias- la pregunta sobre la posibilidad de contar y comunicar lo ocurrido; en los testimonios de Shoah se manifiestan así el historiador Raul Hilberg («It was an authorization to begin something that was not as yet capable of being put into words.»; Lanzmann 1985: 72-73), el superviviente de Chelmno Simon Srebnik («No one can describe it. No one can recreate what happened here. Impossible? And no one can understand it. Even I, here, now»; Lanzmann 1985: 6) o el Sonderkommando Filip Müller («where we were able to tell people, showed that it was of no use, that it made their last moments even harder to bear.»; Lanzmann 1985: 126). También Art Spiegelmann recela de su trabajo frente a su psicoanalista, quien apela al apofatismo en el comienzo del segundo volumen de Maus: «the victims who died can never tell THEIR side of the story, so maybe it's better not to have any more stories» (Spiegelmann 1991: 195). Y, del mismo modo, el reseñista de la reciente Son of Saul cuestiona la validez de las ficciones del Holocausto en The New Yorker:

The question is not one of taste but of imaginative modesty; to watch most feature film-as opposed to documentaries-about the Holocaust [...] is to be left with a lasting moral queasiness about the limits of dramatic reconstruction. Just because you can attempt a thorough depiction of a death camp doesn't mean that you should; if your audience goes away convinced that it now knows what went on at Auschwitz, you've done something wrong. (Lane 2015: 117)

Ante esta perspectiva, como se ha visto, igualmente válida para el testimonio que para su reconstrucción ficcional, hay críticos que hablan de una «retórica de lo indecible» por cuanto lo que no se dice deviene más elocuente que cualquier tentativa de aproximación al horror: «Le silence est tout aussi porteur de sens que la parole» (Antoine 1997). Rosa Lublin, el personaje de Ozick, siente esta impotencia hablando con el comerciante de botones Simon Persky («Whatever I would say, you would be deaf.» [...] Whoever came, they were like deaf people. Whatever you explained to them, they didn't understand»; Ozick 1990: 27), a quien incluso reprocha que su conocimiento del Holocausto obedece solo a las representaciones ficcionales del mismo:

in America cats have nine lives, but we-we're less than cat, so we got three. The life before, the life during, the life after. [...] The life after is now. The life before is our real life, at home, where we was born.»

«And during?»

«This was Hitler.»

«Poor Lublin,» Persky said.

«You wasn't there. From the movies you know it.» (1990: 58; subrayado de la autora)

En su visión de los acontecimientos, la protagonista de «Rosa» adelanta la relación paradójica entre los supervivientes y quienes escuchan su testimonio que ha 
señalado Reyes Mate: «Ellos están atrapados entre la prohibición de callar y la imposibilidad de hablar y, nosotros, entre la necesidad de escucharlos y la imposibilidad de saber» (Mate 2003: 169), de acuerdo con lo que Giorgio Agamben ha denominado «aporía de Auschwitz»:

Por una parte, en efecto, lo que tuvo lugar en los campos les parece a los supervivientes lo único verdadero y, como tal, absolutamente inolvidable; por otra, esta verdad es, en la misma medida, inimaginable; es decir, irreductible a los elementos reales que la constituyen. Unos hechos tan reales que, en comparación con ellos, nada es igual de verdadero. (Agamben 2014: 8-9)

Ya en el «Apéndice de 1976» a Si esto es un hombre Primo Levi daba cuenta de una dificultad inherente a cualquier intento de comprender el horror nazi: «Si comprender es imposible, conocer es necesario, porque lo sucedido puede volver a suceder» (Levi 2011: 242). Es entonces, a juicio de la profesora Fernández Gil, cuando surge la alegoría como vehículo de conocimiento; para contar sin mostrar es preciso utilizar procedimientos de semejanza que evoquen una realidad que escapa a la rigidez del molde de las palabras: «Suggestiveness is basically achieved through the use of a myriad of similes, metaphors and symbols, which permeate the whole story» (Fernández Gil 2012: 64), de acuerdo con las propias palabras de la autora: «Through metaphor, the past has the capacity to imagine us, and we it. [...] Those who have no pain can imagine those who suffer» (Ozick 1991: 283).

Si bien la voz narrativa del primero de los relatos de The Shawl, quien se ve en la tesitura -complicadísima-de contar cómo un guarda del campo de concentración descubre a Magda (hija de Rosa, que había conseguido permanecer oculta hasta ese momento) y la arroja contra una cerca electrificada ${ }^{2}$, se sirve de este entramado retórico de identificaciones, es el silencio lo que, a nivel intradiegético, mantiene con vida a los personajes, tanto al bebé (cuyo único balbuceo transcrito en el relato es un sobrecogedor «Maaaa...aaa!»; Ozick 1990: 8):

Every day Magda was silent, and so she did not die. [...] Magda had been devoid of any syllable; Magda was a mute. Rosa believed that something had gone wrong with her vocal cords, with her windpipe, with the cave of her larynx; Magda was defective, without a voice; perhaps she was deaf; there might be something amiss with her intelligence; Magda was dumb. (1990: 7)

Como a su madre una vez que sobreviene la tragedia. «Tout acte de verbalisation est synonyme de danger» (Antoine 1997):

if she let the wolf's screech ascending now through the ladder of her skeleton break out, they would shoot; so she took Magda's shawl and filled her own mouth with it, stuffed it in and stuffed it in, until she was swallowing up the wolf's screech. (Ozick 1990: 10)

\footnotetext{
${ }^{2}$ La historia parece basarse en una línea de The Rise and Fall of the Third Reich de William L. Shirer (cf. Prose 1989).
} 
Casi de manera complementaria ${ }^{3}$, en «Rosa» es la ilusión por escribirle a una hija a la que imagina todavía viva lo que permite a la protagonista aferrarse a una vida por otra parte carente de sentido: «Too long a time since the last writing. [...] A pleasure, the deeper pleasure, home bliss, to speak in our own language» (1990: 39-40). Aunque otra vez la voz narradora desdice el fantaseo del personaje de ficción: «What a curiosity was to hold a pen [...] all at once this cleanliness, this capacity, this power to make a history, to tell, to explain. To retrieve, to reprieve! To lie» (1990: 44).

Si en las citas aducidas hasta aquí se observan las dificultades derivadas de una posible representación del horror y la enajenación de los supervivientes en los relatos de Ozick, al hablar la autora sobre su propia obra -aun cuando la intentio auctoris no tenga la última palabra sobre lo que un texto puede decir-afirma sin ambages que sus acercamientos al Holocausto deben entenderse solo como artefacto de ficción. En una entrevista ya defendía la necesidad de mantener la distancia con los hechos históricos: «If I felt the weight of history on me, I could never write» (Kauvar y Ozick 1993: 362). Antes aún, en conversación con Francine Prose citada en la reseña del volumen The Shawl, sostenía: «I worry very much that this subject is corrupted by fiction and that fiction in general corrupts history» (Prose 1989). Pero donde Cynthia Ozick muestra mayor grado de desconfianza sobre las ficciones del Holocausto es en este pasaje, esclarecedor:

Sí, todavía estoy preocupada por la corrupción del Holocausto por la literatura de ficción. [...] Yo no tenía ninguna meta al escribir The Shawl. [...] Y no lo considero un testimonio de nada y, de hecho, el relato ni siquiera tiene que ser legitimado porque se trata de ficción... ahora que el Holocausto está siendo declarado una ficción por todo tipo de bastardos. [...] Aunque viviéramos cinco mil años no tendríamos tiempo suficiente para recopilar todos los documentos relacionados con el Holocausto porque en lugar de agotarse, aparecen más y más. (Bonnín 2007: 65)

Estas declaraciones de la autora estadounidense están en consonancia con la crítica de algunos supervivientes, para los que el Holocausto - Auschwitz como su símbolo- habría terminado por convertirse en una especie de parque temático del horror para voyeristas, que termina por hacer que quienes sobrevivieron se sientan como un estorbo por la crudeza no estilizada de su testimonio ${ }^{4}$. Tal era el parecer de Imre Kertész: «More and more often, the Holocaust is stolen from its guardians and made into cheap consumer goods. Or else it is institutionalized [...] with a new and often

${ }^{3}$ No solo en esta oposición triangular silencio-voz-escritura pueden interpretarse como complementarios ambos relatos; en «The Shawl» se reitera la imagen de un infierno helado en el camino al campo de exterminio, mientras que en «Rosa» el calor sofocante convierte a Miami (donde Rosa se retira tras destrozar la tienda de antigüedades que regentaba en Nueva York) en un infierno de signo contrario.

${ }^{4}$ Entre los testimonios de supervivientes tan solo Jorge Semprún parece abogar por el artificio para recrear su experiencia: «Solo alcanzarán esta sustancia [...] aquellos que sepan convertir el testimonio en un objeto artístico, en un espacio de creación. O de recreación» (Semprún 2015: 25). 
phony language» (Kertész 2001: 268). El también superviviente Elie Wiesel se manifestaba con acritud ante la posibilidad de hablar de una «literatura del Holocausto»: «There is no such thing as literature of the Holocaust, nor can there be» (Wiesel 1975: 314). Y señala con disgusto que la materia del horror se ha convertido en un medio de legitimación tan neutro como cualquier otro dentro de un campo cultural, que el paso del tiempo -ya en 1975- ha erosionado la memoria punzante de los supervivientes hasta convertirla en una pasta maleable para ficciones de la industria cultural:

But popularization and exploitation soon followed and then, with the passing of time, it all began to deteriorate. As the subject became popularized, so it ceased to be sacrosanct, or rather was stripped of its mystery. People lost their awe. The Holocaust became a literary «free-for-all,» the «no-man's-land» of modern writing. Now everyone got into the act. Novelists made free use of it in their work, scholars used it to prove their theories, politicians to win votes. In so doing they cheapened the Holocaust; they drained it of its substance. The Holocaust was now a hot topic, fashionable, guaranteed to gain attention and to achieve instant success. (1975: 315)

Si tanto lo que sucedió en los campos como el trauma posterior de los supervivientes solo nos resultan accesibles - que no comprensibles- por la experiencia del testimonio, la vivencia de los campos habría sido para quienes estuvieron allí no tanto indecible o incomunicable como -de nuevo paradójicamente- invivible (cf. Semprún 2015: 25), por cuanto actúa a la manera de un agujero negro, solo permite rastrear la radiación que emite, no ir más allá de su horizonte de sucesos. Esa zona de sombra de la experiencia del horror parece corresponderse con el registro lacaniano de lo Real ${ }^{5}$.

Al combinar la tópica de Lacan con lo narrado en The Shawl puede apreciarse un correlato de los tres campos de la esfera psíquica con lo que le ocurre a Rosa Lublin. Según esta premisa, lo imaginario, «un reino en el que las cosas nos remiten a nosotros mismos» (Eagleton 2010: 20), radicaría en la relación solipsista de Rosa con el chal de Magda, único asidero al recuerdo de su hija asesinada; de ahí su obsesión por recuperarlo -en el segundo de los relatos que componen el volumenpero al mismo tiempo su reticencia a abrir el paquete que lo contendría, que nunca se abre en el espacio de la ficción.

En segundo lugar, la estructura alegórica mencionada en páginas anteriores, el entramado de metáforas y las conexiones semánticas que pueden establecerse entre ellas se correspondería con lo simbólico, un orden que «ha de carecer forzosamente de cimientos» (Eagleton 2010: 147), un armazón donde las relaciones entre sus constituyentes son el sustento de todo el edificio conceptual.

Pero, por último, hay un vacío que escapa a cualquier intento por constreñirlo en palabras. La experiencia del campo supondría la irrupción de lo Real, un punto del que no se puede regresar.

5 Para la aplicación de las dimensiones de lo psíquico a la esfera ética y a los estudios literarios sigo la exposición de Terry Eagleton en Los extranjeros. 
Lo Real es lo que interrumpe esas agradables invenciones, deformando el sujeto y desestructurando el orden simbólico. Es el momento de la crisis y el punto muerto del sujeto, el modo en que no logra ser consigo mismo, [...] la hendidura en nuestro ser por la que fuimos arrancados del cuerpo materno. (Eagleton 2010: 251)

Así lo siente Rosa, para quien pasado y futuro se han teñido de irrealidad tras el contacto con lo Real; el tiempo se le ha transformado en una trama cuyos costurones se le aparecen lo suficientemente burdos como para desenmascarar la ilusión de continuidad. «Before is a dream. After is a joke. Only during stays. And to call it a life is a lie» (Ozick 1990: 58). Por eso, ante los reproches de Persky de que ya no está en el campo de exterminio, de que le basta con mirar la gente que la rodea, ella repone «[w]hat I see is bloodsuckers» (1990: 58). La herida de lo Real le hace soportar a duras penas los parloteos de aquellos que no estuvieron allí.

Aparece entonces el lenguaje no como herramienta, sino como obstáculo, por su incapacidad para comunicar eso que de inaprehensible tiene lo Real. Cabría entonces albergar suspicacias contra la metáfora como instrumento susceptible de generar conocimiento; como ya desmontaba Nietzsche, lo verdadero lo es en la medida en que se lo presenta en una red de relaciones:

¿Qué es entonces la verdad? Una hueste en movimiento de metáforas, metonimias, antropomorfismos, en resumidas cuentas, una suma de relaciones humanas que han sido realzadas, extrapoladas y adornadas poética y retóricamente y que, después de un prolongado uso, un pueblo considera firmes, canónicas y vinculantes; las verdades son ilusiones de las que se ha olvidado que lo son; metáforas que se han vuelto gastadas y sin fuerza sensible. (Nietzsche 1996: 25)

De hecho, para Agamben, «la metáfora se convierte en el reino del lenguaje en lo que el fetiche en el reino de las cosas» (Agamben 2001: 251), lo que vendría a confirmar la denuncia de Elie Wiesel sobre cómo se ha frivolizado el Holocausto.

Pero la existencia es lingüística. La fosilización inherente al cliché o al lugar común solo es superable por renombrar al ser, por poetizar la vivencia. En la modernidad acaso haya sido Hölderlin quien antes advirtió esta ambivalencia del lenguaje, «el más peligroso de los bienes» y al mismo tiempo custodio del ser del ente, porque «lo que queda, lo instauran los poetas» (apud Heidegger 1995: 126).

Eso «que queda», aquello que se resiste a toda formalización y que se puede identificar con lo Real en Lacan, es el testimonio del superviviente:

si el que testimonia verdaderamente de lo humano es aquel cuya humanidad ha sido destruida, eso significa que la identidad entre hombre y no-hombre no es nunca perfecta, que no es posible destruir íntegramente lo humano, que siempre resta algo. El testigo es ese resto. (Agamben 2014:141)

La ficción literaria, por tanto, no puede dar cuenta del Holocausto por la distancia que interpone con el testimonio. Si, como argumenta Reyes Mate, «[e]1 sentido del relato es convertir al oyente en testigo» (Mate 2003: 180), el fracaso de la literatura en la reconstrucción histórica no está reñido con una llamada a la responsa- 
bilidad ética para con las víctimas ${ }^{6}$, mediando entre lo Real y el concepto, acuñando este, pues «lo real debe ser ficcionado para ser pensado» (Rancière 2009: 48); así parecía entenderlo Cynthia Ozick en una mesa redonda, cuando aseguraba que, a priori,

I'm within Theodor W. Adorno's famous dictum: after Auschwitz, no more poetry. And yet, my writing has touched on the Holocaust again and again. I cannot not write about it. It rises up and claims my furies. All the same, I believe that the duty of our generation [...] is to absorb the data, to learn what happened. I am not in favor of making fiction of the data, or of mythologizing or poeticizing it. If we each had a hundred lifetimes, there would not be enough time to assimilate the documents. (apud Juárez Hervás 1994: 363; subrayado del editor)

Quizá no sea un instrumento de conocimiento, pero la ficción sí que puede conducirnos a escuchar la palabra de los supervivientes y evocarla cuando ya no estén, una palabra que «no debe guardar silencio sino guardar al silencio» (Mate 2003: 28).

\section{Referencias bibliográficas}

AgAmBEN, Giorgio (2001). Estancias. La palabra y el fantasma en la cultura occidental. Valencia. Pre-Textos.

- (2014): Lo que queda de Auschwitz. El archivo y el testigo (Homo sacer III). Valencia: Pre-Textos.

AnTOINE, Josée (1997): «Une rhétorique de l'indicible: «The Shawl» de Cynthia Ozick». Journal of the Short Story in English, 28 [en línea]. En: https://jsse.revues.org/79\#text [03/06/2016].

ARISTÓTELES (1999): Poética (edición trilingüe por Valentín García Yebra). Madrid: Gredos. BonNín, Leah (2007): «Una conversación con Cynthia Ozick». Raíces. Revista judía de cultura. 37: 60-66.

Eagleton, Terry (2010): Los extranjeros. Por una ética de la solidaridad. Madrid: Paidós.

FERNÁNDEZ GIL, María Jesús (2012): «Allegorical Traces of the Traumatic in Cynthia Ozick’s The Shawl». ES. Revista de Filología Inglesa 33: 61-80.

HeIDEGGER, Martin (1995): Arte y poesía. Madrid: Fondo de Cultura Económica.

JuÁREZ HERVÁs, Luisa (1994): Judaísmo y feminismo en las historias cortas de Grace Paley y Cynthia Ozick. Tesis doctoral inédita: Universidad Complutense de Madrid.

KaUvar, Elaine M. y OzICK, Cynthia (1993): «An Interview with Cynthia Ozick». Contemporary Literature 34.3: 358-394.

KERTÉSZ, Imre (2001): «Who Owns Auschwitz?» The Yale Journal of Criticism 14.1: 267272.

LANE, Anthony (2015): «Deep and Dark» (reseña Son of Saul). The New Yorker 21 December: 116-117.

${ }^{6}$ A este respecto, me ha resultado muy útil la lectura de «La responsabilidad de la lectura ante el holocausto» de Alberto Sebastián Lago (tesis doctoral inédita, Universidad Carlos III de Madrid, 2012). 
LAnZMAnN, Claude (1985): Shoah: An Oral History of the Holocaust. New York: Pantheon. LEVI, Primo (2011): Trilogía de Auschwitz. Barcelona: El Aleph.

MATE, Reyes (2003): Memoria de Auschwitz. Actualidad moral y politica. Madrid: Trotta. NIETZSCHE, Friedrich (1996): Sobre verdad y mentira en sentido extramoral. Madrid: Tecnos. OzICK, Cynthia (1990): The Shawl. New York: Vintage.

- (1991): Metaphor \& Memory. Essays. New York: Vintage.

Prose, Francine (1989): «Idolatry in Miami» (reseña The Shawl). The New York Times Book Review 10 September: 39 [en línea]. En: http://www.nytimes.com/1989/09/10/books/ idolatry-in-miami.html [06/06/2016].

RANCIÈRE, Jacques (2009): El reparto de lo sensible. Santiago de Chile: LOM.

SEBASTIÁN LAGO, Alberto (2012): La responsabilidad de la lectura ante el holocausto. Tesis doctoral inédita: Universidad Carlos III de Madrid [en línea]. En: http://earchivo.uc3m.es/handle/10016/15214\#preview [28/05/2016].

SEMPRÚn, Jorge (2015): La escritura o la vida. Barcelona: Tusquets.

Spiegelmann, Art (1991): Maus. A Survivor's Tale: II. And Here My Troubles Began. New York: Pantheon.

WIESEL, Elie (1975): «For Some Measure of Humility». Sh'ma: A Journal of Jewish Responsibility 5/100: 314-315. 